\title{
Prevalence of Extended Spectrum $\beta$-Lactamases (ESBLs) Producers Among Gram-Negative Bacilli in Urinary Tract Infections
}

\author{
Abu Hena Md Saiful Karim Chowdhury ${ }^{1 *}$ \\ Md Anwar Husain (Retired) ${ }^{2}$ \\ Nasima Akter ${ }^{2}$ \\ Md Abdul Mazed ${ }^{2}$ \\ Shakeel Ahmed ${ }^{2}$ \\ Tipu Sultan ${ }^{3}$ \\ Arup Kanti Dewanjee ${ }^{4}$ \\ Sanjoy Kanti Biswas ${ }^{5}$ \\ ASM Ashanul Karim ${ }^{6}$ \\ Sheikh Khairul Kabir ${ }^{7}$
}

'Department of Clinical Pathology Chittagong Medical College, Chittagong, Bangladesh.

${ }^{2}$ Department of Microbiology

Chittagong Medical College, Chittagong, Bangladesh.

${ }^{3}$ Department of Virology

Rangamati Medical College, Rangamati, Bangladesh.

${ }^{4}$ Department of Microbiology

Abdul Malek Ukil Medical College, Noakhali, Bangladesh.

${ }^{5}$ Department of Microbiology

Chattagram Maa-O-Shisu Hospital Medical College

Chittagong, Bangladesh.

${ }^{6}$ Department of Medicine

University of Science \& Technology Chittagong (USTC)

Chittagong, Bangladesh

'Department of Medicine

Chittagong Medical College, Chittagong, Bangladesh.

*Correspondence to:

Dr. Abu Hena Md Saiful Karim Chowdhury Clinical Pathologist

Department of Clinical Pathology

Chittagong Medical College, Chittagong, Bangladesh.

Mobile: +8801819636883

E-mail:drsaifulkarim@yahoo.com

www.banglajol.info/index.php/CMOSHMCJ

\begin{abstract}
Background: Antimicrobial resistance is now proclaimed as the most important challenge worldwide being faced by humanity in its fight against infectious diseases. Extended Spectrum $\beta$-Lactamases (ESBLs) producing organisms are increasing in number and causing more severe infections because of their continuous mutation and multidrug resistance property which make its treatment difficult. Aims: The present study was undertaken to detect the prevalence of the ESBLs producing bacteria in urinary tract infection. Methods: Isolated gram-negative bacteria initially screened by Minimum Inhibitory Concentration (MIC) ESBLs breakpoints. Then suspected ESBLs producers were confirmed by phenotypic confirmatory test. Results: 71 $(59.17 \%)$ bacterial strains were isolated from 120 urine samples of patients of suspected urinary tract infection of which $66(92.96 \%)$ were gram-negative and $05(07.04 \%)$ were gram-positive. Among the isolated gram-negative bacteria $63(95.45 \%)$ were found suspected ESBLs producers of which $35(55.56 \%)$ were found as confirmed ESBL producers. The prevalence of ESBLs producing organisms in the present study were found to be $53.03 \%$ and Klebsiella spp. as most prevalent ESBLs producers. Conclusion: It is essential to report ESBL production along with routine sensitivity reporting, which will help the clinician in prescribing the proper antibiotics.
\end{abstract}

Key words : ESBLs; Gram Negative Bacilli; Urinary Tract Infection (UTI); Minimum Inhibitory Concentration (MIC).

\section{INTRODUCTION}

The accelerated emergence of antibiotic resistance among the prevalent pathogens is the most serious threat to the management of infectious diseases ${ }^{1}$. Infections by ESBL producing organisms are causing significant diagnostic and therapeutic problems in affected patient's ${ }^{2}$. ESBLs are mutant forms of $\beta$-lactamases enzymes coded by gens located on transferable plasmids, which can easily spread from one organism to another. The ESBL-producing organisms are often multi-drug resistant, as the plasmids producing ESBLs can carry resistance to other antibiotics ${ }^{3}$.

Extended spectrum $\beta$-lactamases producing bacteria produce ESBL enzymes that mediate resistance to extended spectrum (third generation) cephalosporins (e.g. ceftazidime, cefotaxime, ceftrixone etc.) and monobactams (e.g. aztreonam) but do not affect cephamycins (e.g. cefoxitin and cefotatan) or carbapenems (e.g. meropenem or imipenem) and are inhibited by -lactamase inhibitors such as clavulanate, sulbactam and tazobactam ${ }^{1,4,5}$.

The ESBL-producing bacteria are increasingly causing Urinary Tract Infection (UTI) becoming a major threat for patients in the hospital, long-term care facilities and community. The increasing drug resistance among these bacteria has made therapy of UTI difficult and has led to a greater use of expensive broad-spectrum antibiotics ${ }^{6}$. Inappropriate antibiotic selection in infections caused by these organisms is associated with treatment failures, poor clinical outcomes, prolonged hospital stay, increased morbidity, mortality and health care costs. 
ESBLs have spread threateningly in many regions of the world and they presently comprise over 300 variants. The widespread use of the third generation cephalosporins and aztreonam is believed to be the major cause of the mutations in these enzymes, which has led to the emergence of the ESBLs ${ }^{7}$ Drug resistance of this form is often difficult to recognize by using conventional antimicrobial susceptibility methods. Failure to identify ESBL producing organisms also contributes to their uncontrolled spread. Therefore, identification of the resistant phenotypes is important, particularly in developing countries where there is excessive use of antibiotics and lack of adequate antimicrobial resistance surveillance ${ }^{8}$.

This study was designed to investigate the prevalence of ESBLs producing organisms among uropathogens which would guide clinicians and microbiologists for proper handling of these pathogens \& prevent unnecessary use of antibiotics.

\section{MATERIALS \& METHODS}

This cross sectional study was carried out in the department of Microbiology, Chittagong Medical College, during the period of June 2008 to May 2009. Samples were collected after taking informed written consent from both sexes and different age groups patients of indoor and outpatient department of Chittagong Medical College Hospital, Chittagong.

Inclusion criteria: Patients with clinical signs/symptoms of urinary tract infection.

Exclusion criteria: Pus cell $<10 / \mathrm{HPF}$ in a centrifuged urine sample 9 .

Using all aseptic precautions clean-catch mid-stream urine samples about $15-20 \mathrm{ml}$ were collected in sterile, wide mouth, tightly closed, leak-proof container by standard technique-for microscopy, culture and sensitivity test. After inoculating in Cystine Lactose Electrolyte Deficient (CLED) agar media by calibrated wire loop $(0.01 \mathrm{ml})$, identification of organisms were done as per standard laboratory methods of identification. A specimen was considered positive for UTI if a single organism was cultured at a concentration of $10^{5}$ Colony Forming Unit/ml. Antimicrobial sensitivity of the isolates were tested by Kirby-Bauer disc diffusion technique against different antimicrobial agents, except imipenem, ceftriaxone, ceftazidime and cefotaxime which were tested for MIC by agar plate dilution method.

ESBL Detection: The method recommended by Clinical Laboratories Standard Institute (CLSI) requires a two-step approach of initially screening for ESBL production and then performing confirmatory tests on screen positive isolates ${ }^{10}$.

Screening for ESBL producers by dilution method

Agar dilution method: The screening for ESBL producers was done by agar dilution method as was recommended by CLSI. Any of the isolated organisms found to be grown at this stated screening antibiotics concentration (that is, MIC of the ceftriaxone, ceftazidime and cefotaxime $>2 \mathrm{~g} / \mathrm{ml}$ ) according to CLSI, 2007 was considered as possible ESBL producers and spelled for the confirmatory tests. The use of more than one antimicrobial agent for screening improves the sensitivity of detection $^{10}$.
Detection of ESBLs by the confirmatory tests

Phenotypic confirmatory test: Confirmation of the ESBLproducing isolates was done by the phenotypic confirmatory test according to CLSI recommendation. In this test, third generation cephalosporin i.e. ceftazidime $(30 \mu \mathrm{g})$ and cefotaxime $(30 \mu \mathrm{g})$ disc alone and in combination with clavulanic acid $(10 \mu \mathrm{g})$ were used. Ceftazidime, cefotaxime discs without clavulanic acid were placed on one side and ceftazidime, cefotaxime discs combined with clavulanic acid $(30 / 10 \mu \mathrm{g})$ were placed on other side of the inoculated plate. After overnight incubation at $37^{\circ} \mathrm{C}$, diameter of zone of inhibition was measured. A $5 \mathrm{~mm}$ or more increases in diameter of zone of inhibition for ceftazidime and cefotaxime tested in combination with clavulanic acid versus its zone when ceftazidime and cefotaxime tested alone confirms an ESBLs producing organism ${ }^{10}$

Reference strain for quality control used for ESBL detection

E. coli BB-32327 (CTX-M9) was used as positive control and E. coli ATCC (American Type Culture Collection) 25922 was used as negative control of ESBL detection test.

\section{RESULTS}

A total 120 urine samples from patients of suspected urinary tract infection were studied, of which 71 $(59.17 \%)$ bacterial strains were isolated (Table-1).

Table 1 : Frequencies of urinary pathogens.

\begin{tabular}{lcc} 
Culture Results & Number & Percentage \\
Culture Positive & 71 & 59.17 \\
Culture Negative & 49 & 40.83 \\
Total & 120 & 100.0 \\
\hline
\end{tabular}

Among the 71 bacterial isolates, of which majority were E. coli 45(63.38\%), followed by Klebsiella species 14(19.72\%), Enterococci species 05(07.04), Acinetobacter species 03(04.22\%), Proteus species 02(02.82\%) \& Pseudomonas species 02(02.82\%) (Table-2).

Table 2 : Distribution of bacterial species among the isolates $(n=71)$.

Name of bacterial species Number $(n=71) \quad$ Percentage

Gram-negative organism

$\begin{array}{lll}\text { E. coli } & 45 & 63.38\end{array}$

Klebsiella species

14

Acinetobacter species

Proteus species

03

Pseudomonas species

Total

66

Gram-positive organism

Enterococci species

Grand Total

71

19.72

04.22

02.82

02.82

92.96

07.04 100.00

It appears from Table- 3 that total 66 isolated gram-negative bacteria were screened for suspected ESBLs producers on the basis of MIC breakpoints by agar dilution method, out of which $63(95.45 \%)$ were found suspected ESBLs producers \& $03(4.55 \%)$ gave negative result. 
Table 3 : Detection of ESBL producing bacteria on the basis of MIC (screening test) by agar-dilution method $(n=66)$.

\begin{tabular}{lcc}
$\begin{array}{c}\text { ESBL by agar-dilution } \\
\text { method }\end{array}$ & $\begin{array}{c}\text { Number of } \\
\text { isolates }\end{array}$ & Percentage (\%) \\
\hline Positive & 63 & 95.45 \\
Negative & 03 & 04.55 \\
Total & 66 & 100.0 \\
\hline
\end{tabular}

Screening positive 63 spected ESBLs producing bacteria were subjected to phenotypic confirmatory test, 35(55.56\%) were found as confirmed ESBL producers \& $28(44.44 \%)$ showed negative result (Table-4).

Table 4 : Detection of ESBL producing organisms on the basis of phenotypic confirmatory test $(n=63)$.

\begin{tabular}{lcc}
$\begin{array}{l}\text { Phenotypic } \\
\text { confirmatory test }\end{array}$ & $\begin{array}{c}\text { Number of } \\
\text { isolates }\end{array}$ & Percentage (\%) \\
Positive & 35 & 55.56 \\
Negative & 28 & 44.44 \\
Total & 63 & 100.0 \\
\hline
\end{tabular}

Out of 66 gram-negative bacteria 35(53.03\%) were found to ESBLs producer. Higher rate of ESBLs was observed in Klebsiella species 08 (57.14\%) out of 14 , followed by E. coli $24(53.33 \%)$ out of 45 , Proteus species $01(50.00 \%)$ Pseudomonas Species 01(50.00\%) \& Acinetobacter species $01(33.33 \%)$ out of 03 (Table-5).

Table 5 : Distribution of ESBLs producers among gram-negative bacteria $(n=66)$.

\begin{tabular}{lcc} 
Name of bacteria & $\begin{array}{c}\text { Total no. of gram- } \\
\text { negative bacteria }\end{array}$ & $\begin{array}{c}\text { Number of ESBL } \\
\text { producers }\end{array}$ \\
\hline E. coli & 45 & $24(53.33)$ \\
Klebsiella species & 14 & $08(57.14)$ \\
Acinetobacter species & 03 & $01(33.33)$ \\
Proteus species & 02 & $01(50.00)$ \\
Pseudomonas species & 02 & $01(50.00)$ \\
Total & 66 & $35(53.03)$ \\
\hline
\end{tabular}

- Figures within parentheses indicate percentages

\section{DISCUSSION}

In the present study, a total of 120 urine samples from patients of different age groups were collected from which (culture positive) urinary isolates were $71(59.17 \%)$. This result is closely related to that of Rahman in BSMMU, Dhaka, who found $53.57 \%$ culture positive isolates from urine samples ${ }^{11}$. Among the bacterial isolates, 66(92.96\%) were gram-negative and $05(07.04 \%)$ were gram-positive in our study (Table-2). Similar to present study Rahman and Alim of BSMMU, Dhaka found $90 \%$ gram-negative \& $10 \%$ gram-positive and $90.21 \%$ gram-negative $\& 9.79 \%$ gram-positive isolates respectively ${ }^{11,12}$.
Amongst the isolates in our study, E. coli (63.38\%) was the highest urinary isolates followed by Klebsiella (19.73\%), Enterococci (7.04\%), Acinetobacter (4.22\%), Pseudomonas $(2.82 \%) \&$ Proteus spp. $(2.82 \%)$. Our results are also in agreement with those of Rahman and Alim in BSMMU, Dhaka who also found E. coli followed by Klebsiella spp. as the most prevalent urinary isolates ${ }^{11,12}$. Most common agents causing UTIs are gram negative bacilli. E coli, a normal flora of gastrointestinal tract, is the commonest causative organism of UT I ${ }^{13}$.

The reason of high ratio for E. coli is the presence of this bacteria in the feces, thus it cause autoinfection. In addition, after gaining entry to the bladder, E. coli are able to attach to the bladder wall and form a biofilm that resists the body's immune response $\mathrm{e}^{14}$.

As of now, no country wide study has been conducted for the detection of the prevalence of ESBL production in Bangladesh, individual studies which were done in different parts of the country showed a varying prevalence, based on various risk factors and local reasons.

In the present study, we found 63(95.45\%) suspected ESBLs producers from 66 gram-negative isolates, based on MIC, ESBLs screening breakpoints (Table-3). As using more than one antibiotic increase the sensitivity, we used three third generation cephalosporins (ceftriaxone, ceftazidime \& cefotaxime) for the screening ${ }^{10}$. Our finding is closely related to that of Metri et al. in North Karnataka, India, who found 91.74\% suspected ESBLs producers by screening test ${ }^{7}$. When these 63 screening positive isolates were subjected to the confirmatory tests, $35(55.56 \%)$ were confirmed as ESBL producers by Phenotypic Confirmatory Test (PCT) (Table-4).

The prevalence of ESBLs producing organisms in the present study were found (Table-5) to be $53.03 \%$, which is higher than that of Alim 23.19\% and Rahman 30.90\% both in BSMMU but lower than that of Biswas of BSMMU $80 \%$ and Yasmin of Mymensingh $71.30 \%$. Ullah et al. (2009), in Pakistan found $58.7 \%$ ESBL producers ${ }^{11,12,15,16}$. The prevalence of ESBLs producers in India ranges from $6.6 \%$ to $91 \%$, in Europe from $23-25 \%$ for Klebsiella spp. and $5.4 \%$ for E. coli and in United States from 0 to $25 \%$, depending on the institution ${ }^{3,7}$.

The variation on ESBL positivity might be due to the number of isolates studied, variation in institution to institution, geographic location and also country to country ${ }^{5,8}$. The prevalence of ESBL production is high in the referral centers and the intensive care unites where the patients are referred from the peripheral centers and where the antibiotic use is profuse $^{7}$. The higher prevalence compared to western countries can be explained by the fact that western countries have strict infection control policies and practices, efficient and effective antibiotic audit systems, shorter average hospital stays, better nursing barriers, and other important health care measures which substantially decrease the chances of acquisition and spread of ESBLs strains. The uncontrolled use of 3rd generation cephalosporins at our hospital could be a leading contributory factor to the high ESBLs prevalence observed in this study ${ }^{17}$. 
ESBLs are most commonly recognized in Klebsiella spp. and E. coli and most prevalent in Klebsiella pneumoniae ${ }^{18,19}$. We also found Klebsiella spp. (57.14\%), as the leading ESBLs producers followed by E. coli (53.33\%), Proteus (50.00\%), Pseudomonas spp .01 (50.00\%) and Acinetobacter spp. $(33.33 \%)$ in our study, which correlates with those of Alim \& Rahman in BSMMU, Yasmin at Mymensingh, Metri et al. \& Giriyapur et al. in India who also found Klebsiella spp. as the most common ESBL producers ${ }^{7,8,10,11,16}$.

The high occurrence of ESBLs in Klebsiella spp. is of great concern since infections caused by this bacterium are very common and resistance of the organism may be due to the presence of capsule that gives some level of protection to the cells, presence of multidrug resistance efflux pump, easy spreading nature, pathogenic and efficient at acquiring and disseminating resistance plasmid.
Klebsiella spp. has some virulence factor like hyper-viscosity, polysaccharide capsule and production of endotoxin, carbapenemases, which make it more resistant $\mathrm{t}^{20,21}$.

\section{CONCLUSION}

Existing of extended spectrum $\beta$-lactameses in bacteria and their potential multidrug resistance will create serious problems in the future as their continuous mutation and limited therapeutic option. Indiscriminate use of antibiotics especially 3rd generation cephalosporins and monobactams should be avoided. The regular detection of ESBLs producing organisms should be carried out in every laboratory.

\section{DISCLOSURE}

All the authors declared no competing interest.

\section{REFERENCES}

1. Al-Jasser AM. Extended-spectrum $\beta$-lactamases (ESBLs): a global problem. Kuwait Med J. 2006; 38: 171-185.

2. Livermore DM. $\beta$-lactamases in laboratory and clinical resistance. Clinical Microbiology Reviews. 1995; 8(4): 557-584.

3. Sirot D. Extended-spectrum plasmid mediated beta-lactamases. J Antimicrob Chemother. 1995; 36 (Suppl A): 19-34.

4. Paterson DL, Bonomo RA. Extended-spectrum $\beta$-lactamases: a clinical update, Clinical Microbiology Reviews. 2005; 18(4):657-686.

5. Bradford PA. Extended-spectrum $\beta$-lactamases in the $21^{\text {st }}$ century: characterization, epidemiology, and detection of this important resistant threat. Clinical Microbiology Reviews. 2001; 14(4), 933-951.

6. Kader AA, Angamuthu K.Islam KMS. Extended-spectrum $\beta$-lactamases in urinary isolates of Escherichia coli, Klebsiella pneumoniae and other gram-negative bacteria in a hospital in Eastern Province, Saudi Arabia. Saudi Med J. 2005; 26(6): 956-959.

7. Metri BC, Jyothi P, Peerapur BV. The prevalence of ESBL among Enterobaceriaceae in a tertiary care hospital of North Karnataka, India. J of Clinical and Diagnostic Research. 2011; 5(3): 470-475.

8. Kader AA, Kumar A, Krishna A, Zaman MN. An Accelerated method for the detection of Extended-spectrum $\beta$-lactamases in urinary isolates of Escherichia coli, Klebsiella pneumoniae. Saudi J Kidney Dis Transpl. 2006; 17(4): 535-539.

9. Sobel JD, Kaye D. Urinary Tract Infections in GL Mandell, JE Bennett \& R Dolin (eds), Mandell, Doglus and Bennett's principles and practice of infectious diseases, Churchill Livingstone, Philadelphia. 2010;(7):957-985.

10. Clinical and Laboratory Standards Institute. Performance standards for antimicrobial susceptibility testing, seventeenth informational supplement. CLSI Document M100-S17, Wayne, Pennsylvania, USA. 2007; 27(1): 1-177.

11. Rahman M. Rapid detection of extended-spectrum $\beta$-lactamases production directly from primary culture. M. Phil. Thesis, Bangabandhu Sheikh Mujib Medical University, Dhaka. 2007.

12. Alim R. Detection of extended-spectrum- $\beta$-lactamases (ESBLs) producing bacteria. M. Phil. Thesis, Bangabandhu Sheikh Mujib Medical University, Dhaka. 2005.

13. Svanborg C, Godaly G. Bacterial virulence in urinary tract infection. Infect Dis Clin North AM. 1997; 11:513-529.

14. Khamees SS. Urinary Tract Infection: causative agents, the relation between bacteriuria and pyuria. World Applied Sciences Journal. 2012; 20(5): 683-686.

15. Biswas SM. Comparison of three dimensional test and double disc synergy test for detection of extended spectrum $\beta$-lactamase (ESBL) producing gram-negative bacteria. M.Phil. Thesis, Bangabandhu Sheikh Mujib Medical University, Dhaka. 2009.

16. Yasmin T. Prevalence of ESBL among Esch. coli and Klebsiella spp. in a tertiary care hospital and molecular detection of important ESBL producing genes by multiplex PCR. M.Phil. Thesis, Mymensingh Medical College, Mymensingh. 2012.

17. Ahamed K, Thokar MA, Toboli AS, Fomda BA, Bashir G, Maroof P. Extended spectrum- $\beta$-lactamase mediated resistance in Escherichia coli in a tertiary care hospital in Kashmir, India. African J of Microbiology Rasearch. 2010; 4(24): 2720-2728.

18. Bush K, Jacoby GA, Medeiros AA. A functional classification scheme for $\beta$-lactamases and its correlation with molecular structure. Antimicrobial Agents and Chemotherapy. 1995; 39(6): 1211-1233.

19. Tribuddharat C, Srifuengfung S, Chiangjong W. A correlation between phenotypes and genotypes of extended-spectrum beta-lactamase (ESBL) - producing Klebsiella pneumoniae in a university hospital, Thailand. J Infect Dis Antimicrob Agents. 2007; 24(3): 117-123.

20. Yushau MM, Kumurya AS, Suleiman L. Prevalence of extended spectrum $\beta$-lactamases among Enterobacteriaceae in Murtala Mohammed specialist hospital, Kano,N. J of Pure and Applied Sciences. 2010; 3(1): 169-172.

21. Lin Y, Lu M, Tang H, Liu H, Chen C, Liu K, et al. Assessment of hypermuco-viscosity as a virulence factor for experimental Klebsiella pneumoniae infections: comparative virulence analysis with hypermuco-viscosity-negative strain. BMC Microbiology. 2011; 11(50) viewed on 15 April 2012, http://www.biomedcentral.com/1471-2180/11/50. 